REVISTA PROYECCIONES N॰ 8: 109-136

Diciembre 1984 - ISSN 0716-0917

JORNADA MATEMATICAS, Agosto 1984.

\title{
USO DE COMPONENTES PRINCIPALES EN DATOS COMPOSICIONALES
}

JORGE GALBIATI RIESCO*

\section{EL PROBLEMA DE LAS COMPONENTES PRINCIPALES.}

Cuando se dispone de datos multidimensionales, es deseable reducir su dimensionalidad, a cambio de perder una parte de la información. Esto es debido a que es muy difícil representar y entender la información contenida en datos de dimensiones mayores que 2 ó 3 . La reducción de dimensionalidad puede hacerse de diferentes formas, apuntando todas ellas a que la pérdida de información, por la simplificación que se hace, sea mínima.

En el análisis en componentes principales, se centra la atención en la varianza, pues una mayor varianza permite separar más las observaciones y así poder discriminar mejor a los individuos que confor-

* Profesor Departamento Matemáticas, Universidad Católica de Valparaiso. 
man la muestra. Desde este punto de vista, la varianza viene a ser una medida de la cantidad de información disponible. Cuando en alguna dimensión hay una varianza pequeña, los datos se agrupan en lo que puede considerarse un sólo conglomerado, en esa dimensión, la que tiende a ser desechada del análisis, por aportar poca información.

Supongamos que se tiene un vector aleatorio $\mathrm{x}, \mathrm{p}$-dimensional, esperanza $\mathrm{E}(\mathrm{X})=\mu$, matriz de varianzas-covarianzas $\operatorname{var}(\mathrm{X})=\sum=\left(\delta_{i j}\right)$. El vector $\mathrm{X}$ representa la información p-dimensional. Convencionalmente losvectores $\mathrm{V}$ como columnas, en términos de sus coordenadas.

Interesa encontrar una dirección en el espacio $R^{p}$, tal que la proyección de $\mathrm{x}$ sobre ella tenga varianza máxima.

Es decir, se debe encontrar un vector $A=\left(A_{1}, A_{2}, \ldots, A_{p}\right)$, tal

que

$Y=A^{\prime} X$

tenga varianza máxima, sujeto a la condición de normalización

$A^{\prime} A=1$

para independizar el resultado de la magnitud de A.

Se tiene que

$\operatorname{Var}(\mathrm{Y})=\operatorname{Var}\left(\mathrm{A}^{\prime} \mathrm{X}\right)=\mathrm{A}^{\prime} \operatorname{Var}(\mathrm{X}) \mathrm{A}=\mathrm{A}^{\prime} \sum \mathrm{A}$

Entonces se debe maximizar $A^{\prime} \sum A$, sujeto a la condición $A^{\prime} A=1$.

Derivando la función

$\Phi(A, \lambda)=A^{\prime} \sum A+\lambda\left(1-A^{\prime} A\right)$ 
$\Phi(A, \lambda)=\sum_{k, 1} A_{k} \delta{ }_{k 1} A_{1}+\lambda\left(1-\sum_{k} A_{k}^{2}\right)$

respecto de los componentes de A e igualando a cero las derivadas para encontrar puntos críticos, se obtiene lo siguiente, considerando la simetría de $\Sigma$ :

$\frac{\partial \Phi}{\partial A_{j}}=\sum_{1} \delta_{j 1} A_{1}+\sum_{k} A_{k} \delta_{k j}-2 \lambda A_{j}=0 \quad(j=1,2, \ldots \ldots p)$

$\frac{\partial \Phi}{\partial A_{j}}=2 \sum_{1} \delta_{j 1} A_{1}-2 \lambda A_{j}=0$

En forma vectorial, se puede expresar

$\frac{\partial O}{\partial \dot{A}}=2 \sum A-2 \lambda A=0$

Derivando respecto del multiplicador de Lagrange $\lambda$,

$\frac{\partial \Phi}{\partial \lambda}=1-\sum_{k} A_{k}^{2}=0$

De la ecuación (5),

$\Sigma \mathrm{A}=\lambda \mathrm{A}$

La varianza, entonces, es

$\operatorname{Var}(\mathrm{Y})=\mathrm{A}^{\prime} \sum \mathrm{A}=\lambda \mathrm{A}^{\prime} \mathrm{A}=\lambda$

\section{AIGUNAS PROPIEDADES MATRICIATESS.}

DERINICION.

Dada una matriz $\Sigma$, un vector no nulo $v$ tal que cumple la ecua ción

$\Sigma \mathrm{v}=\lambda \mathrm{v}$ 
en que $\lambda$ es un escalar, se denomina vector propio de $\Sigma$. El escalar $\lambda$ se llama valor propio de $\sum$.

Definición: Sea $\Sigma$ una matriz simétrica real de orden $\mathrm{p} \times \mathrm{p}$

a) $\quad \sum$ es semidefinida positivassi dado $v \in R^{p}, v \neq 0$, se cumple que $v^{\prime} \Sigma v \geqslant 0$.

b) $\quad \sum$ es definida positivassi dado $v$ e $R^{p}, v \neq 0$, se cumple que $\mathrm{v}^{\prime} \sum \mathrm{v}>0$.

Proposición: Sea $\Sigma$ una matriz simétrica real. Entonces sus valores pro pios son reales.

Proposición: Sea $\sum$ una matriz simétrica, real, de orden p x p. $\sum$ tiene $p$ vectores propios, que forman una base ortogonal de $\mathbb{R}^{p}$, si $\sum$ no sing.

Proposición: Los valores propios de una matriz definida positiva son po sitivos.

Proposición: Si $\sum$ es una matriz simétrica real de orden $\mathrm{p} x \mathrm{p}$, existe una matriz ortogonal $\alpha$ tal que

$\alpha^{\prime} \Sigma \alpha=D$

$\operatorname{con} D=\operatorname{diag}\left\{\lambda_{1}, \lambda_{2}, \ldots \ldots, \lambda_{p}\right\}$

Los $\lambda_{i}$ son los valores propios de $\sum y \alpha=\left[a_{1}, a_{2}, \ldots, a_{p}\right]$, matriz cuyas columnas son los vectores propios de $\Sigma$, ordenados de acuer do a los valores propios en D.

Definición: Si dos matrices A y B están relacionadas por una ecuación del tipo

$\mathrm{U}^{-1} \mathrm{AU}=\mathrm{B}$

para alguna matriz U no singular, se dice que son similares. 
En nuestro caso $\Sigma$ es matriz similar a una matriz diagonal $D$. Se dice entonces, que $\sum$ esdiagonizable. Además se da la particularidad de que la matriz simétrica es diagonizable a través de una matriz ortogonal, pues $\alpha$ es ortogonal $\left(\alpha^{\prime} \alpha=\alpha \alpha^{\prime}=I\right)$

Proposición: Si $\sum$ y D se relacionan de acuerdo a la ecuación $\alpha^{\prime} \sum \alpha=D$, entonces

$\operatorname{traza}(\Sigma)=\operatorname{traza}(D)=\sum_{1=1}^{p} \quad \lambda_{i}$

Y

$\operatorname{det}(\Sigma)=\operatorname{det}(D)=\prod_{1=1}^{p} \lambda_{i}$

\section{SOLUCION AL PROBLEMA DE LAS COMPONENTES PRINCIPALES.}

Supondremos que la matriz de varianza-covarianza $\sum$ es definida positiva, por construcción.

De acuerdo con la definición, y por la ecuación (7), se ve que la solución al problema de las componentes principales está dada por los vectores propios normalizados (de norma 1) de la matriz de varianza-covarianza $\Sigma$.

Como las varianzas obtenidas son los correspondientes valores propios (8), queda como solución aquel vector propio correspondiente al mayor de los valores propios, y que simbolizaremos $A_{1}$ y $\lambda_{1}$ respectivamente.

Corresponde a un máximo absoluto, pues si $\mathrm{V}$ es otro vector no nulo de $\mathbb{R}^{p}$, de norma $1, y$ como los vectores propios $A_{1}, A_{2}, \ldots, A_{p}$ for man una base ortogonal, $\mathrm{V}$ puede expresarse como combinación lineal de ellos: 
$V=\sum_{I=1}^{p} c_{i} \quad A_{i}$

$\mathrm{V}$ tiene norma 1 , luego

$I=V^{\prime} V=\sum_{i=1}^{p} \sum_{j=1}^{p} \quad c_{i} c_{j} A_{i} A_{j}=\sum_{i=1}^{p} \quad C_{i}^{2}$

por la ortogonalidad de los $\mathrm{A}_{i}$.

Entonces $\operatorname{Var}\left(V^{\prime} X\right)=V^{\prime} \operatorname{Var}(X) V=V^{\prime} \sum V=\sum_{i=1}^{p} \quad c_{i} A ! \quad \sum_{j=1}^{p} c_{j} A_{j}=$

$=\sum_{i=1}^{p} \sum_{j=1}^{p} \quad C_{i} C_{j} A_{i} \quad \sum A_{j}=\sum_{i=1}^{p} \sum_{j=1}^{p} c_{i} C_{j} A_{i} \quad \sum A_{j}=$

$=\sum_{i=1}^{p} \sum_{j=1}^{p} c_{i} c_{j} \lambda_{i} \quad A_{i} \quad A_{j}=\sum_{i=1}^{p} c_{i}^{2} \lambda_{i}$

por la ortogonalidad de los $A_{i}$.

De (10) y (11) resulta que $\operatorname{Var}\left(V^{\prime} X\right)$ es un promedio ponderado de $\operatorname{los} \lambda_{i}$, cuyas ponderaciones $\mathrm{C}_{i}^{2}$ suman 1 , por 10 que es menor $\circ$ igual que el mayor de los $\lambda_{i}, \lambda_{1}$.

Por 10 tanto este último es un máximo absoluto de la función $\mathrm{Y}=\mathrm{A}^{\prime} \mathrm{X}$ sujeto a la restricción $\mathrm{A}^{\prime} \mathrm{A}=1, \mathrm{Y}$ se alcanza este máximo cuando $A$ es igual al vector propio $A_{1}$ asociado a él.

\section{EL SISTRMA DE ENES PRINCIPALES.}

\section{DEFINICION.}

La proyección del vector aleatorio x sobre el vector $A_{1}$, solución del problema de las componentes principales, se denomina Primera Componente principal. La dirección en el espacio $\mathbb{R}^{\mathrm{P}}$ definida por $\mathrm{A}_{1}$, se denomina Primer Eje Principal. 
Hemos visto que la varianza de $\mathrm{x}$ proyectado sobre $A_{1}$, $\mathrm{Y}=\mathrm{A}^{\prime}{ }_{1} \mathrm{X}$, es $\mathrm{A}^{\prime}{ }_{1} \sum \mathrm{A}_{1}$, en que $\sum$ es la matriz de varianza-cova rianza de $X$. Y es la máxima sobre las varianzas de todas las proyecciones posibles de $x$ sobre vectores de norma 1 .

A continuación supondremos los vectores propios $A_{1}$ ' $A_{2}, \ldots . . A_{p}$ ordenados según el orden de sus respectivos valores propios $\lambda_{1}, \lambda_{2}, \ldots, \lambda_{p}$, de mayor a menor.

Definición: Dado un vector aleatorio $x$, su k-ésima componente principal es su proyección sobre el vector propio normali zado correspondiente al valor propio de orden $k$, ordenados de mayor a menor $(1 \leqslant k \leqslant p)$. El k-ésimo eje principal es la di rección definida por este vector.

Proposición: Sean $A_{1}, A_{2}, \ldots . ., A_{p}$ los vectores propios de $x$, ordenados de acuerdo a sus respectivos valores propios en or den de mayor a menor. Sea $k$ un entero tal que $1 \leqslant k \leqslant p$, y sea $\mathrm{s}_{\mathrm{k}}$ el subespacio de $\mathbb{R}^{\mathrm{P}}$ generado por los vectores propios $\mathrm{A}_{\mathrm{k}}$, $A_{k+1}, \ldots ., A_{p}$.

Las proyecciones de $x$ sobre un vector normalizado de $\mathrm{s}_{\mathrm{k}}$ tiene máxima varianza cuando ese vector es $\mathrm{A}_{\mathrm{k}}$.

La demostración es similar a la demostración de que la varianza máxima se obtiene con $A_{1}$, haciendo las sumas des de $k$ hasta $p$.

\section{ESTRUCTURA DE VARIANZAS.}

Las componentes principales, entonces, definen direc ciones ortogonales en las que al proyectar el vector aleatorio $x$, se obtiene varianzas máximas, cada una ortogonal a las restantes. Con esto se forma un nuevo sistema de coorde 
nadas cuyas direcciones, en el espacio original, están dadas por $A_{1}, A_{2}, \ldots ., A_{p}$, en ese orden. Los valores de las varian zas son respectivamente $\lambda_{1}, \lambda_{2}, \ldots ., \lambda_{p}$, en orden decrecien te de magnitud.

La matriz que transforma las coordenadas de los vectores del sistema de ejes a los ejes principales es $\mathbb{A}^{\prime}$, en que

$$
\mathbb{I A}^{\prime}=\left[\begin{array}{c}
\mathrm{A}_{1}^{\prime} \\
\mathrm{A}_{2}^{\prime} \\
\vdots \\
\mathrm{A}_{\mathrm{p}}^{\prime}
\end{array}\right]
$$

cuyas filas son los vectores propios.

En efecto, si $V$ es un vector de $\mathbb{R}^{p}$, en las coordenadas principales $V$ se expresa

$$
\mathbb{A} \cdot V=\left[\begin{array}{l}
A_{1}^{\prime} V \\
A_{2}^{\prime} V \\
\vdots \\
A_{p}^{\prime} V
\end{array}\right]
$$

siendo cada coordenada las respectiva componente principal.

Además IA es precisamente la matriz ortogonal que diagonaliza $\Sigma$, pues por estar constituida por vectores propios,

$\sum I A=D I A, \operatorname{con} D=\operatorname{diag}\left\{\lambda_{1}, \lambda_{2}, \ldots ., \lambda_{p}\right\}$

y como es ortogonal,

$I A \cdot \sum I A=D$ 
La varianza total de $x=\left(x_{1}, x_{2}, \ldots ., x_{p}\right)$, es

$\sum_{i=1}^{p} \operatorname{var}\left(x_{i}\right)=\sum_{i=1}^{p} \quad \delta_{i i}=\operatorname{traza}(\Sigma)$

Si $Y=I A^{\prime} X=\left[\begin{array}{l}A_{1}^{\prime} X \\ A_{2}^{\prime} X \\ \vdots \\ A_{p}^{\prime} X\end{array}\right]$

las coordenadas especiales, la varianza total de Y es

$\sum_{i=1}^{p} \operatorname{var}\left(A_{1}^{\prime} X\right)=\sum_{i=1}^{p} \quad \lambda_{i}=\operatorname{tr}(D)$

La proporción de varianza de la componente principal k (varianza explicada por esa componente) es

$\frac{\operatorname{var}\left(A_{k}^{\prime} X\right)}{\operatorname{varianza\operatorname {total}}}=\frac{\lambda_{k}}{\lambda_{1}+\lambda_{2}+\ldots+\lambda_{p}}=\frac{\lambda_{k}}{\operatorname{traza}(D)}$

La proposición de varianza explicada por las primeras $k$ componentes principales es

$\frac{\lambda_{1}+\lambda_{2}+\ldots+\lambda_{k}}{\lambda_{1}+\lambda_{2}+\ldots+\lambda_{p}}$

Como $\lambda_{1} \geqslant \lambda_{2} \geqslant \ldots \geqslant \lambda_{p}$, dada una proposición de varian $z a \quad q 1$, se puede encontrar un número entero $k \leqslant p$ tal que

$\frac{\lambda_{1}+\lambda_{2}+\ldots+\lambda_{k-1}}{\operatorname{tr}(D)}<q \leqslant \frac{\lambda_{1}+\lambda_{2}+\ldots+\lambda_{k}}{\operatorname{tr} \text { (D) }}$ 
Si $\mathrm{k}<\mathrm{p}$, el subespacio generado por las primeras $\mathrm{k}$ com ponentes principales explica al menos la proporción q de la varianza total del vector aleatorio $\mathrm{Y}$ ( $\circ \mathrm{X}$ ).

En tal caso se ha logrado reducir la dimensión de la variable aleatoria que representa la información, con lo que se ha logrado simplificar el problema. El costo ha sido una pérdida de información, que en todo caso se ha minimizado, y que no es mayor que la fracción 1-q de la varianza total. Se ha minimizado precisamente porque las componentes principales han maximizado la varianza proyectada en los ejes.

\section{ESTRUCTURA DE COVARIANZAS.}

La matriz de varianzas-covarianzas de $x$ es $\Sigma$, por 10 que $\operatorname{cov}\left(x_{i}, x_{j}\right)=\delta_{i j}$, el elemento $(i, j)$ de la matriz.

La covarianza entre las proyecciones sobre dos ejes princip les distintos es

$\operatorname{cov}\left(y_{i}, y_{j}\right)=\operatorname{cov}\left(A_{i}^{\prime} x, A_{j} x\right)=A_{i}^{\prime} \operatorname{Cov}\left(x, x^{\prime}\right) A_{j}=A_{i}^{\prime} \operatorname{var}(x) A_{j}=$ $=A_{i}^{\prime} \sum A_{j}=\lambda_{j} A_{i}^{\prime} A_{j}=0$

si $i \neq j$, por ortogonalidad.

Este resultado, de que las covarianzas entre proyecciones de $x$ sobre los ejes principales es cero, ya se podía haber deducido, pues

$$
\begin{aligned}
& \operatorname{Var}(Y)=\operatorname{Var}\left(\mathbb{I} A^{\prime} X\right)=\mathbb{I} A^{\prime} \operatorname{Var}(X) \mathbb{I A}=\mathbb{I A} \cdot \sum \mathbb{I A} \\
& \operatorname{Var}(Y)=D=\operatorname{diag}\left\{\lambda_{1}, \lambda_{2}, \ldots, \lambda_{\mathrm{P}}\right\}
\end{aligned}
$$


Por último, la covarianza entre $\mathrm{X}$ e $\mathrm{Y}$ es

$\operatorname{Cov}(X, Y)=\operatorname{Cov}\left(X^{\prime}, I A^{\prime} X\right)=\operatorname{Cov}\left(X^{\prime} X\right) I A=\operatorname{Var}(X) I A=\sum I A$

Pero $I A \cdot \sum I A=D Y I A$ es ortogonal; luego

$\sum I A=I A D$

$\operatorname{COV}(X, Y)=\operatorname{IAD}$

Con lo que

$\operatorname{cov}\left(x_{i}, y_{j}\right)=(\operatorname{IAD})_{i j}=A_{i j} \lambda_{j}$

La correlación es

$$
\begin{aligned}
& \operatorname{corr}\left(x_{i}, y_{j}\right)=\frac{A_{i j} \lambda_{j}}{\sqrt{\operatorname{var}\left(x_{i}\right) \operatorname{var}\left(y_{j}\right)}}=\frac{A_{i j} \lambda_{j}}{\sqrt{\delta_{i i} \lambda_{j}}} \\
& \operatorname{corr}\left(x_{i}, y_{j}\right)=A_{i j} \sqrt{\frac{\lambda_{j}}{\delta_{i i}}}
\end{aligned}
$$

\section{SituACION MUESTRAL.}

Ahora supongamos que disponemos de una muestra de $n$ observaciones provenientes de un vector aleatorio $\mathrm{x}$ p-dimensional.

Usaremos el símbolo X para denotar la matriz de datos de orden nxp, cuyas filas $x_{k}=\left(x_{k_{1}}, x_{k 2}, \ldots x_{k p}\right)$ son las observaciones p-dimensionales y las columnas $x_{(j)}$ las variables - caracteristicas medidas. supondremos $n>p$. 
La matriz de varianzas-covarianzas $\sum$ se estima por la matriz de varianzas-covarianzas muestral,

$s_{p x p}=\frac{1}{n} \sum_{k=1}^{n}\left(x_{k}-\bar{x}\right)\left(x_{k}-\bar{x}\right)^{\prime}=\frac{1}{n} \sum_{k} x_{k} x_{k}^{\prime}-\bar{x} \bar{x} \cdot=\left(s_{i j}\right)$

$\operatorname{con} \bar{x}_{p \times 1}=\frac{1}{n} \sum_{k=1}^{n} \quad x_{k} \quad$ vector de medias

Las propiedades que se refieren a valores propios se mantienen, pues s es simétrica, semidefinida positiva, aunque se supondrá que es definida positiva, pues este es el caso normal. Para que no 10 sea, tendría que tener rango menor que p. Esto significa que las columnas de $x$ son linealmente dependientes, 10 que es muy poco probable por ser una muestra aleatoria, por lo tanto es razonable suponer que no ocurra esta situación.

Las componentes principales, en forma natural, se es timan utilizando los vectores propios normalizados $a_{i}$ de $S$, $y$ se ordenan de acuerdo al orden descendente de magnitud de sus respectivos valores propios $1_{i}$.

En tal caso se dan las siguientes propiedades:

sea $\alpha=\left[a_{1}, a_{2}, \ldots . a_{p}\right]$ la matriz cuyas columnas son los vectores propios de s ordenados según los valores pro pios.

Entonces $\alpha^{\prime} \mathrm{s} \alpha=\mathrm{L}$

$\operatorname{con} L=\operatorname{diag}\left\{1_{1}, 1_{2}, \ldots, 1_{p}\right\}$

y a ortogonal.

L es la matriz de varianzas-covarianzas de la matriz 
de datos transformados al nuevo sistema de coordenadas principales.

$\not=\not \alpha$

$\Downarrow=\left(y_{i j}\right)$

y ijepresenta la magnitud de la proyección de la observación i-ésima $x_{i}$ sobre el j-ésimo vector propio $a_{j}$.

Por lo tanto la varianza de la j-ésima variable de Y es $1_{j}$, y la covarianza entre dos variables distintas es cero.

La proporción de varianza contenida en la proyección de la variable j-ésima es

$\frac{1_{j}}{1_{1}+1_{2}+\ldots+1_{p}}=\frac{1_{j}}{\operatorname{traza}(L)}$

y la proporción de la varianza contenida en las proyecciones de las $k$ primeras variables es

$\frac{1_{1}+1_{2}+\ldots+1_{k}}{1_{1}+1_{2}+\ldots+1_{p}}$

La covarianza entre $\Varangle$ e $\$$ está dada por la matriz de covarianza

$\operatorname{Cov}(x, x)=\frac{1}{n} \sum_{i}\left(x_{i}-\bar{x}\right)\left(y_{i}-\bar{y}\right)^{\prime}=\frac{1}{n} \sum_{i} x_{i} y_{i}-\bar{x} \bar{y}$

en que $x_{i}$ e $y_{i}$ son las filas i-ésimas de $k$ e $\not$ respectivamen te, $y$

$\bar{x}=\frac{1}{n} \sum_{i=1}^{n} x_{i} \quad$ e $\quad \bar{y}=\frac{1}{n} \sum_{i=1}^{n} y_{i}$ 
vectores de medias.

$$
\text { Pero } x=x \alpha \text { luego }
$$

$\operatorname{Cov}(X, Y)=\frac{1}{n} \sum_{k=1}\left(x_{k}-\bar{x}\right)\left(x_{k}-\bar{x}\right)^{\prime} \alpha=s \alpha$

Además $\alpha$ 's $\alpha=\mathrm{I}$ y $\alpha$ es ortogonal, luego

$\mathrm{s} \alpha=\alpha{ }^{\prime} \mathrm{L}$

La covarianza entre una variable original $x_{\text {(i) }} y$ otra variable $y_{(j)}$ en un eje principal, es entonces

$\operatorname{cov}\left(x_{(i)}, y_{(j)}\right)=\left(\alpha^{\prime} L\right)_{i j}=a_{i j} 1_{j}$

su correlación es

$$
\begin{aligned}
& \operatorname{corr}\left(x_{(i)} y_{(j)}\right)=\frac{a_{i j}{ }^{1_{j}}}{\sqrt{\operatorname{var}\left(x_{(i)}\right) \operatorname{var}\left(y_{(j)}\right)}}= \\
& =\frac{a_{i j}{ }^{1}}{\sqrt{s_{i i}{ }^{1} j}}=a_{i j} \sqrt{\frac{1_{j}}{s_{i i}}}
\end{aligned}
$$

\section{COMPONENTES PRINCIPALES GENERALIZADAS.}

Tiene por lo tanto, el inconveniente de preservar la forma que tiene el conjunto de datos en el espacio $\mathbb{R}^{\mathrm{P}}$. Si el 
conjunto presenta algún tipo de curvatura, una transformación lineal entregará coordenadas que no podrán adaptarse a ella, perdiéndose la posibilidad de reducir aún más la dimensionalidad de la información.

Esto sí se puede lograr utilizando coordenadas curvilíneas adecuadas. El procedimiento es el siguiente:

El vector aleatorio original es

$$
x=\left[\begin{array}{c}
x_{1} \\
x_{2} \\
\vdots \\
x_{p}
\end{array}\right]
$$

y se dispone de un conjunto de $\mathrm{k}$ funciones conocidas (posiblemente no li neales) de $x, f_{1}(x), f_{2}(x), \ldots, f_{k}(x), k \geqslant p$, linealmente independientes. $k$ no debe ser menor que $p$, con el objeto de preservar la cantidad de información.

Entonces se dispone de un nuevo vector aleatorio

$$
F=\left[\begin{array}{l}
f_{1}(x) \\
f_{2}(x) \\
\vdots \\
f_{k}(x)
\end{array}\right]
$$

al cual se le aplicará un análisis en componentes principales como el in dicado anteriormente. Es decir, se buscarán vectores ortogonales normalizados $A_{1}, A_{2}, \ldots ., A_{k}$, tales que las combinaciones lineales

$$
\begin{aligned}
& A_{i}^{\prime} F=\sum_{j=1}^{k} a_{i j} f_{j}\left(x_{1}, x_{2}, \ldots, x_{p}\right) \\
& i=1,2, \ldots, k
\end{aligned}
$$


tengan máxima varianza el primero, y máxima varianza cada uno de los siguientes en el subespacio ortogonal a los anteriores vectores propios $\mathrm{A}_{i}$.

Como en el caso lineal, estos vectores son los vectores propios de la matriz varianzas-covarianzas de $F, \sum_{F}$.

En el caso de una muestra, se trabaja en forma análoga, transformando primero los datos, y luego efectuando el análisis como se indica en el punto 7 .

\section{DATOS COMPOSICIONALES.}

Los datos composicionales son vectores de proporciones que componen un todo. Por lo tanto tienen la restricción de que sus coordenadas suman 1 .

Por ejemplo, en geología, las proporciones de diversos compuestos determinados, que forman un tipo de roca, constituyen un dato composicional. La composición, en proporciones de nacionalidades de un grupo humano. Las proporciones de respuestas por alternativas en una pregunta de alternativas múltiples, en una encuesta.

Veamos algunas definiciones y notaciones relacionadas con datos composicionales:

Designaremos $\mathbb{P}^{\mathrm{P}}$ al octante positivo de $\mathbb{R}^{\mathrm{p}}$,

$$
\mathbb{P}^{\mathrm{P}}=\left\{\left(\mathrm{x}_{1}, \mathrm{x}_{2}, \ldots \ldots, \mathrm{x}_{\mathrm{p}}\right) / \mathrm{x}_{i}>0, i=1,2, \ldots, \mathrm{p}\right\}
$$

Designaremos $S^{P}$ al simplex positivo de $\mathbb{I}^{p}$,

$$
s^{p}=\left\{\left(x_{1}, x_{2}, \ldots ., x_{p}\right)^{\prime} / x_{1}+x_{2}+\ldots .+x_{p}<1, x_{i}>0, i=1,2, \ldots p\right\}
$$


Y designaremos $\mathbb{H}^{\mathrm{p}+1}$ a la región del octante positivo de $\mathbb{R}^{\mathrm{p}}$ del hiperplano cuya ecuación es $x_{1}+x_{2}+\ldots \ldots+x_{p}=1$, $\mathbb{H}^{p+1}=\left\{\left(x_{1}, x_{2}, \ldots, x_{p+1} / x_{1}+x_{2}+\ldots+x_{p+1}=1, x_{i}>0, i=1,2, \ldots, p+1\right\}\right.$

\section{DEFINICION.}

Un vector o punto de $\mathbb{H}^{\mathrm{p}+1}$ se llamará composición, y un conjunto de tales vectores, datos composicionales.

Se usará la notación $\mathrm{x}^{(\mathrm{p})}$ para enfatizar la dimensión de $\mathrm{x}$.

Dado un vector $x^{(p)}$ e $s^{p}, x^{(p)}=\left(x_{1}, x_{2}, \ldots, x_{p}\right)^{\prime}$, se puede cons truír una composición en forma natural, $x^{(p \neq 1)} \in \mathbb{H}^{p+1}$ haciendo $x_{p+1}=1-$ $x_{1}-x_{2}-\ldots-x_{p}, y x^{(p+1)}=\left(x_{1}, x_{2}, \ldots, x_{p}, x_{p+1}\right)^{\prime}$, el vector $x^{(p)} a u-$ mentado agregando la coordenada $x_{p+1}$.

$$
\begin{aligned}
& \text { Si } c<p, x^{(c)}=\left(x_{1}, x_{2}, \ldots, x_{c}\right)^{\prime} \text { es un subvector de } x^{(p)}=\left(x_{1},\right. \\
& \left.x_{2}, \ldots . x_{p}\right)^{\prime} \text {. } \\
& T\left(x^{(c)}\right) \text { denotará la suma } T\left(x^{(c)}\right)=x_{1}+x_{2}+\ldots+x_{c}
\end{aligned}
$$$$
\text { Consideremos la función }
$$$$
C: \mathbb{P}^{\mathrm{p}+1} \rightarrow \mathrm{s}^{\mathrm{p}} \text { definida por }
$$$$
x^{(p)}=C\left(W^{(p+1)}\right) \text { en que }
$$$$
x_{i}=\frac{w_{i}}{T\left(w^{(p+1)}\right)} \quad \frac{w_{i}}{w_{1}+w_{2}+\ldots+w_{p+1}} \quad i=1,2, \ldots, p
$$

Definición: El vector $W^{(p+1)}$ tal que $\left.x^{(p)}=C W^{(p+1)}\right)$ se llama base de $x^{(p)}$. 
De la definición anterior, se ve claramente que dado un vector $x^{(p)}$, su base no es única. En efecto, si $w$ es una base de $x$, bW también 10 es, en que b es un escalar positivo cualquiera.

\section{DIFICULTADES EN EL TRATAMIENTO DE DATOS COMPOSICIONALES.}

Los datos composicionales presentan dos características que es necesario hacer notar.

La primera es que la suma de sus coordenadas es 1 . Esta restricción implica que pertenecen a un subespacio de dimensión menor en una unidad añ número de coordenadas. Por 10 tanto la matriz de varianzas-covarianzas tiene rango una unidad menor que el número de filas.

El análisis en componentes principales busca producir ejes orto gonales sobre los cuales las proyecciones de la variable aleatoria tengan máximas varianzas en direcciones ortogonales. Con esto último se busca la no coorrelación entre las componentes.

Sin embargo, cuando se trata de proporciones, no es una ventaja encontrar correlaciones nulas, puesto que ellas naturalmente están corre lacionadas (con tendencia a ser éstas negativas), por su naturaleza.

La segunda característica se presenta con frecuencia en datos composicionales, aunque no en general. Consiste en posibles curvaturas mostradas por conjuntos de estos datos. Como se dijo antes, un simple análisis en componentes principales dá una transformación lineal de los datos, por 10 que las coordenadas que se obtienen no pueden adaptarse a las curvaturas eventuales.

Los datos composicionales $\mathrm{x}^{(\mathrm{p}+1)}$ se suelen tratar de tres distintas maneras:

La primera es hacerlo tal como vienen, como $x^{(p+1)}$, con la restricción que la suma de sus componentes es 1 . 
El resultado es que la matriz de varianzas-covarianzas es singu lar, de rango p. Por lo tanto tiene un valor propio cero. Su correspon diente vector propio tiene coordenadas iguales, lo cual es consecuencia de que la suma de las coordenadas de $\mathrm{x}$ es 1 . Por lo tanto los demás, por ser ortogonales con él, son contrastes o comparaciones lineales. Luego las p componentes principales con varianza mayor que cero, son con trastes de proporciones de un mismo total. La interpretación de las correlaciones nulas resulta difícil., por tratarse precisamente de proporciones.

La segunda forma es desechando una componente, elegida arbitrariamente, transformándose el vector de $\mathrm{x}^{(\mathrm{p}+1)} \in \mathbb{H}^{\mathrm{p}+1}$ a un vector $\mathrm{x}_{-j}^{(\mathrm{p})} \in$ $S^{p}$, en que $x_{-j}$ es el vector $x$ al cual se le ha eliminado la j-ésima coor denada. Sin embargo siempre existe una restricción sobre el vector, por lo que no se soluciona el problema de la interpretación; la restricción, ahora, es que la suma es menor que 1. Además el resultado será dependien te de la elección que se haga de la componente a eliminar.

La tercera forma de tratar datos composicionales es mediante la transformación

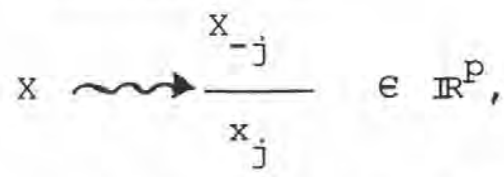

en que se ha eliminado la coordenada $x_{j}$, escogida arbitrariamente, $y$ se ha dividido las demás por ella. El resultado también dependerá de la va riable eliminada, y la transformación mantiene la linealidad que no permite manejar las curvaturas adecuadamente.

\subsection{IAA TRANSFORMACION LOG - RAZON.}

La transformación propuesta por J. Aitchison para el tratamiento de datos composicionales es

$F: \mathbb{H}^{\mathrm{p}+1} \longrightarrow \mathbb{R}^{\mathrm{p}}$ en que 
$F=\left[\begin{array}{l}f_{1}(x) \\ f_{2}(x) \\ \vdots \\ f_{p}(x)\end{array}\right]$ $\operatorname{con} f_{i}(x)=f_{i}\left(x_{1}, x_{2}, \ldots, x_{p+1}\right)=\left(\log \frac{x_{1}}{x_{j}}, \log \frac{x_{2}}{x_{j}}, \ldots, \log \frac{x_{j-1}}{x_{j}}, \log \frac{x_{j+1}}{x_{j}}, \ldots\right.$ $\log$ $x_{j}$

$F(x)=\log \left(\frac{x_{-j}}{x_{j}}\right)$

en que $\mathrm{xe} \mathbb{H}^{\mathrm{p}+1}$ y $\mathrm{x}_{-j}$ es el vector $\mathrm{x}$ al que se le ha omitido la coordenada $x_{j}$.

La matriz de varianzas-covarianzas de los datos transformados es

$\Omega_{j}=\operatorname{Var}(F(x))=\operatorname{Var}\left(\log \frac{x_{-j}}{x_{j}}\right)$

La no-linealidad de la función logarítmica permite manejar la posible curvatura de los datos. Subsiste el problema de la asimetría, pues distintas elecciones del divisor $\mathrm{x}_{\mathrm{j}}$ conducirán a distintas componentes principales.

Sea $A^{*}=\left(a_{1}^{*}, a_{2}^{*}, \ldots, a_{p}^{*}\right)$ ' uno de los vectores que definen los ejes principales. La correspondiente componente principañ del vector $F$ es

$$
\sum_{i=1}^{p} a_{i}^{* f} f_{i}^{p+1} \sum_{\substack{i=1 \\ i \neq j}}^{p} \quad a_{i} \log \left(\frac{x_{i}}{x_{j}}\right)
$$


con

$$
\begin{aligned}
& a_{k}= \begin{cases}a_{k}^{*} & \text { si } 1 \leqslant k<j \\
a_{k-1}^{*} & \text { si } j<k \leqslant p\end{cases} \\
& \text { Si se define } a_{j}=-\sum_{\substack{i=1 \\
i \neq j}}^{p+1} a_{i} \text {, entonces }
\end{aligned}
$$

Iuego

$$
\sum_{\substack{i=1 \\ i \neq j}}^{p+1} a_{i} \log \left(\frac{x_{i}}{x_{j}}\right)=\sum_{i=1}^{p+1} \quad a_{i} \text { log } x_{i}
$$

O sea, los componentes principales en este caso pueden ser expre sadas simétricamente en términos de contrastes log-lineales de las p+1 proporciones, cualquiera sea la elección del divisor $x_{j}$. En todo caso, debe recordarse que los $a_{i}$ dependen del $x_{j}$ que se ha escogido. 


$$
\begin{aligned}
& \text { Además, como por (39) } \sum_{i=1}^{p+1} a_{i}=0 \text {, entonces } \\
& \left(\begin{array}{cc}
p+1 & \\
\sum_{i=1} & a_{i}
\end{array}\right)\left(\begin{array}{cc}
p+1 & \log x_{j} \\
\sum_{j=1} & p+1
\end{array}\right)=0 \\
& y \sum_{i=1}^{p+1} a_{i} \log x_{i}=\sum_{i=1}^{p+1} a_{i} \log x_{i}-\sum_{i=1}^{p+1} a_{i} \sum_{j=1}^{p+1} \frac{\log x_{j}}{p+1}= \\
& =\sum_{i=1}^{p+1} a_{i}\left(\log x_{i}-\sum_{j=1}^{p+1} \frac{\log x_{j}}{p+1}\right)= \\
& =\sum_{i=1}^{p+1} a_{i}\left[\log x_{i}-\log \left(x_{1}, x_{2}, \ldots, x_{p+1}\right)^{\frac{1}{p+1}}\right]= \\
& =\sum_{i=1}^{p+1} a_{i}\left[\log \frac{x_{i}}{\left(x_{1}, x_{2}, \ldots, x_{p+1}\right)^{1 / p+1}}\right]
\end{aligned}
$$

Luego

$$
\sum_{i=1}^{p} a_{i}^{* f} f_{i}=\sum_{i=1}^{p+1} a_{i} \log x_{i}=\sum_{i=1}^{p+1} a_{i} \log \left\{\frac{x_{i}}{g(x)}\right\}
$$

$\operatorname{con} g\{x\}=\left(x_{1}, x_{2}, \ldots . x_{p+1}\right)^{1 / p+1}$, el medio geométrico de las $p+1$ proporciones $\mathrm{x}_{i}$.

Esto sugiere que pareciera ser conveniente estudiar los valores y vectores propios de la matriz de varianzas-covarianzas de orden p+1.

$$
\Omega=\operatorname{var} \log \left\{\frac{x^{(p+1)}}{g(x)}\right\}=\left(\Omega_{k 1}\right)
$$

con

$$
\Omega_{k 1}=\operatorname{cov}\left(\log \frac{x_{k}}{g(x)}, \log \frac{x_{1}}{g(x)}\right)
$$


$\Omega$ es semidefinida positiva, por construcción (es matriz de varianzas-co varianzas).

Estudiemos su posible valor propio nulo:

Sea $A=\left(a_{1}, a_{2}, \ldots, a_{p+1}\right)^{\prime}$ el vector propio asociado a él. En tonces

$\Omega A=0$

$\mathrm{p}+1$

$\sum_{1=1} \Omega_{\mathrm{k}_{1}{ }^{a} 1}=0 \quad \mathrm{k}=1,2, \ldots \ldots, \mathrm{p}+1$

$\sum_{1=1}^{p+1} \operatorname{cov}\left(\log \frac{x_{k}}{g(x)}, \log \frac{x_{1}}{g(x)}\right) a_{1}=0$

pero

$\sum_{1=1}^{p+1} \operatorname{cov}\left(\log \frac{x_{k}}{g(x)}, \log \frac{x_{1}}{g(x)}\right) a_{1}=$

$=\operatorname{cov}\left(\log \frac{x_{k}}{g(x)}, \sum_{1=1}^{p+1} a_{1} \log \frac{x_{1}}{g(x)}\right)=$

$=\operatorname{cov}\left(\log \frac{x_{k}}{g(x)}, \log \frac{x_{1}^{a 1}, x_{2}^{a 2}, \ldots, x_{p+1}^{a p+1}}{g(x)^{p+1}}\right)$

Entonces si $a_{1}=a_{2}=\ldots=a_{p+1}=1$, queda

$\operatorname{cov}\left(\log \frac{x_{k}}{g(x)}, \log \frac{g(x)^{p+1}}{g(x)^{p+1}}=\right.$

$\operatorname{cov}\left(\log \frac{x_{k}}{g(x)}, \log 1\right)=\operatorname{Cov}\left(\log \frac{x_{k}}{g(x)}, 0\right)=0$ 
pio cero.

Luego $\mathbb{1}_{p+1}=(1,1, \ldots, 1)^{\prime}$ es un vector asociado al valor pro

Entonces se buscará un máximo de p soluciones de

$\Omega A=\lambda A \quad$ o bien

$\left(\Omega-\lambda I_{p+1}\right) A=0$

$\operatorname{con} A_{1}, A_{2}, \ldots, A_{p}$ vectores de norma 1 .

Al ser ortogonales con el vector propio 1 , son todos contrastes log-lineales de las proporciones $x_{i}$, como se quería.

Si consideramos la matriz

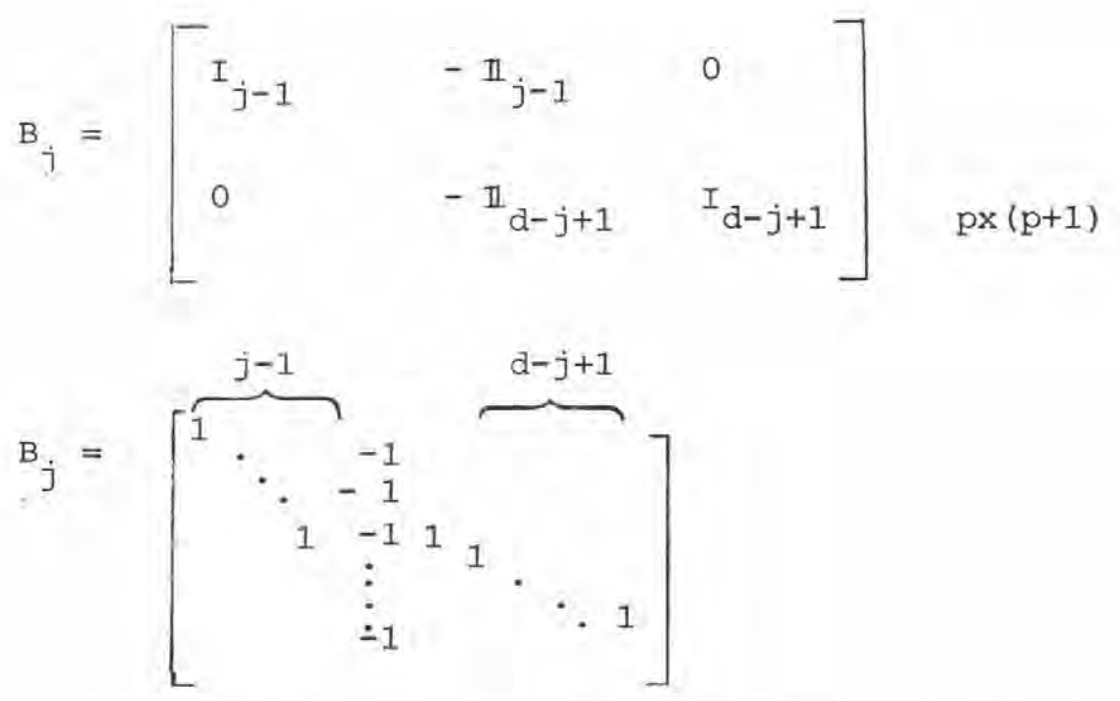

Se establece la relación entre la matriz de varianza-covarianza $\Omega_{j}$ (37) y la matriz $\Omega$ de (46),

$\Omega_{j}=B_{j} \Omega_{j} B_{j}$

Premultiplicando (46) por $\mathrm{Bj}$, 
$\left(B_{j} \Omega-\lambda B_{j}\right) \quad A=0$

y observando que

$A=B_{j}^{\prime} A_{-j}$, en que $A_{-j}$ es $A_{j}$

al que se le ha eliminado la coordenada j-ésima, queda

$$
\begin{aligned}
& \left(B_{j} \Omega B_{j}^{\prime}-\lambda B_{j} B_{j}^{\prime}\right) A_{-j}=0 \\
& \left(\Omega_{j}-\lambda H_{p}\right){ }_{-j}=0
\end{aligned}
$$

o en general,

$\left(\Omega_{j}-\mu \mathrm{H}_{\mathrm{p}}\right) \quad E=0$

con $\mu$ y E incógnitas y con

${ }_{p}^{H}=B_{j} B_{j}^{\prime}=\left[\begin{array}{ccccc}1 & 2 & \ldots & \ldots & 2 \\ 2 & 1 & \ldots & \ldots & 2 \\ \vdots & \vdots & \ddots & & \\ \vdots & \vdots & \ddots & \ddots & \\ \vdots & \vdots & & \ddots & \\ 2 & 2 & & & 1\end{array}\right]$

Entonces el problema simétrico (46) y el problema asimétrico

(49) producen componentes principales log-lineales relacionadas por las ecuaciones.

$\left.\begin{array}{l}A=B_{j}^{\prime} \quad E \\ E=A_{-j}\end{array}\right\}$ 
Los vectores propios de la versión asimétrica $E_{1}, E_{2}, \ldots, E_{p}$ no son ortogonales, pero satisfacen las relaciones

$E_{i}^{\prime} H_{d} E_{j}=\left\{\begin{array}{lll}1 & \text { si } & i=j \\ 0 & \text { si } & i \neq j\end{array}\right.$

\section{TECNICAS DE ANALISIS CON LA TRANSFORMACION LOG-RAZON.}

La ecuación (49) proporciona un análisis en componentes principales que difiere del usual, en que sus ejes no son ortogonales, sino que responden a las relaciones definidas por (51). Tiene las ventajas sobre los procedimientos utilizados usualmente, y que se describieron anteriormente, de que no es lineal por lo que se puede adaptar a las posibles curvaturas, $y$ de que los datos no están restringidos, sino que se mueven en $\mathbb{R}^{\mathrm{p}+1}$. Aún subsiste, eso si, el problema de la asimetría, pues el resultado dependerá de la coordenada que se elimine.

Pero se relaciona con el análisis proporcionado por (46), a tra vés de la ecuación (48), o de las relaciones entre vectores propios (50). Estos indican que los vectores propios del problema asimétrico (49) no son sino las proyecciones de los vectores propios del problema simétrico (46), sobre el subespacio que resulta de eliminar la respectiva coordena da que se seleccionó. El problema simétrico (46) tiene las ventajas que señalamos sobre el problema asimétrico, pero además es indiferente con respecto a las coordenadas, sin que deba privilegiarse ninguna de ellas, haciendo el resultado dependiente de cuál se ha escogido. Es todo esto lo que hace atractiva la utilización de la transformación log-razón con un conjunto de datos composicionales.

El procedimiento es el siguiente: 
Se efectúa la transformación de los datos de acuerdo a (36). Lue go se calcula la matriz de varianzas-covarianzas $\Omega$, y se buscan los valores y vectores propios proporcionados por (46). Estos últimos definen los ejes principales. Como en el análisis de componentes principales usual, los valores propios $1_{i}$, corresponden a los valores de las varianzas de las proyecciones respectivas. La proporción de varianza explicada por las primeras $\mathrm{k}$ componentes, también es

$1_{1}+1_{2}+\ldots+1_{k}$

$1_{1}=1_{2}+\ldots+1_{p+1}$ 


\section{BIBLIOGRAFIA}

1)

J. AITCHISON, S.M. SHEN.

"Logistic-normal Distributions. Some Properties and Uses". Biometrika, Vol. $67, \mathrm{~N}^{\circ} 2,1980$.

2)

J. AITCHISON.

"A New Approach to Null Correlations of Proportions". Mathematical GeologY, Vol. 13, $\mathrm{N}^{\circ} 2,1981$.

3)

J AITCHISON.

"The Statistical Analysis of Composi tional Date". J.R. Statistical Socie ty B, Vol. $44, \mathrm{~N}^{\circ} 2,1982$.

4) J. AITCHISON.

"Principal Component Analysis of Com positonal Data". Biometrika, Vol. 70 $\mathrm{N}^{\circ} 1,1983$.

5) R. GNANADESIKAN.

"Methods for Statistical Data Analysis of Multivariate observations". Ed. John Wiley, 1977.

6) P. GREEN, J.D. CARROL.

"Mathematical Tools for Applied Multivariate Analysis". Ed. Academic Press, 1976.

7) K.V. MARDIA, J.T. KENT, "Multivariate Analysis". Ed. Academic J.M. BIBBY. Press, 1979. 